Pacific Journal of Mathematics

THE HAUSDORFF DIMENSION OF A SET OF NORMAL

w Douglas Pollingto 


\title{
THE HAUSDORFF DIMENSION OF A SET OF NORMAL NUMBERS
}

\author{
A. D. Pollington
}

\begin{abstract}
Suppose that numbers $2,3, \cdots$ are partitioned into two disjoint classes $R, S$ so that rational powers lie in the same class. In this paper we prove that the set of numbers $\xi$ which are normal to every base from $R$ and to no base from $S$ has Hausdorff dimension 1 . The existence of such numbers was first shown by W. M. Schmidt.
\end{abstract}

1. Introduction. We call two natural numbers $r, s$ equivalent and write $r \sim s$, when each is a rational power of the other.

Schmidt [2] has shown that normality to base $r$ implies normality to base $s$ precisely when $s$ is a rational power of $r$ and also [3] that, given any partition of the numbers $2,3, \cdots$ into two disjoint classes $R, S$ so that equivalent numbers fall in the same class, there are real numbers normal to every base from $R$ and to no base from $S$.

In this paper we prove the following.

TheOREM 1. Given any partition of the numbers $2,3, \cdots$ into two disjoint classes $R, S$ so that equivalent numbers fall in the same class, the set, $\mathscr{N}$, of numbers which are normal to every base from $R$ and to no base from $S$ has Hausdorff dimension 1.

If $R$ is empty then $\mathscr{N}$ consists of those numbers which are not normal to any integer base. In this case Theorem 1 is already known, see for example Schmidt [4]. If $S$ is empty then $\mathscr{N}$ consists of those numbers which are normal to all integers bases. This set contains almost all numbers, in the sense of Lebesgue's measure, and Theorem 1 is obvious. We will therefore restrict our attention to the case when $R=\left\{r_{1}, r_{2}, \cdots\right\}$ and $S=\left\{s_{1}, s_{2}, \cdots\right\}$ are both nonempty.

After some preliminaries, and given a certain parameter $A$, a nested sequence

$$
J_{0}=[0,1] \supset J_{1} \supset \cdots
$$

of sets is constructed, where each set $J_{i}$ is a union of closed intervals. It is then shown that a number

$$
\xi \in \bigcap_{i=1}^{\infty} J_{i}
$$

is nonnormal to each base $s_{1}, s_{2}, \cdots$. Then a new sequence of sets

$$
K_{0}=[0,1] \supset K_{1} \supset \cdots
$$


is constructed, where each $K_{i} \subseteq J_{i}$, and it is shown that a number

$$
\xi \in \bigcap_{i=1}^{\infty} K_{i}
$$

is normal to each base $r_{1}, r_{2}, \cdots$. For this, estimates of exponential sums and two lemmas of Schmidt [3] are required. Finally, a theorem of Eggleston [1] is used to show that $\bigcap_{i=1}^{\infty} K_{i}$ has Hausdorff dimension at least $\log (A-1) / \log A$. Since $A$ can be chosen arbitrarily large, the desired conclusion follows.

We will require the following lemma, due to Schmidt [3], which is the cornerstone of his proof that $\mathscr{N}$ is nonempty.

Lemma 1. Let $K, l, r, s$ be natural numbers with $l \geqq s^{K}$ and $r \nsim s$. Then

$$
\sum_{n=0}^{N-1} \prod_{k=K+1}^{\infty}\left|\cos \left(\pi r^{n} l / s^{k}\right)\right| \leqq 2 N^{1-\alpha(r, s)} \quad \text { where } \quad \alpha(r, s)>0 .
$$

The following result implies Theorem 1.

TheOREM 2. Let $A>2$ be a natural number. Let $R, S$ be two subsets of $\{A, A+1, \cdots\}$ such that if $r \in R$ and $s \in S$ then $r \propto s$. Then the set $\mathscr{N}_{A}$ of numbers which are normal to every base from $R$ and to no base from $S$ has Hausdorff dimension at least $\log (A-1) / \log A$.

2. Deduction of Theorem 1 from Theorem 2. Suppose that we are given a partition of the natural numbers $R, S$ as in Theorem 1. Let $R_{A}=R \cap\{A, A+1, \cdots\}, S_{A}=S \cap\{A, A+1, \cdots\}$.

We apply Theorem 2 for $R_{A}, S_{A}$. Then $\mathscr{N}_{A}=\mathscr{N}$. For suppose $r \in R$ and $x \in \mathscr{N}_{A}$. Then clearly if $r \geqq A$ then $x$ is normal to base $r$, if $r<A$, then $r^{A}>A$ and also $r^{A} \in R$ since rational powers lie in the same class. Hence $x$ is normal to base $r^{A}$. But then $x$ is also normal to base $r$. Similarly $x$ is nonnormal to base $s$ for any $s \in S$.

Hence $\mathscr{N}_{A} \subset \mathscr{N}$ and clearly $\mathscr{N} \subset \mathscr{N}_{A}$. Thus

$$
\bigcup_{A=3}^{\infty} \mathscr{N}_{A}=\mathscr{N}
$$

But

$$
\operatorname{dim}\left(\bigcup_{A=3}^{\infty} \mathscr{N}_{A}\right) \geqq \frac{\log (A-1)}{\log A} \quad A=3,4, \cdots .
$$

Thus $\operatorname{dim} \mathscr{N}=1$ which proves Theorem 1. 
We now construct a subset of $\mathscr{N}_{A}$ to show that

$$
\operatorname{dim} \mathscr{N}_{A} \geqq \frac{\log (A-1)}{\log A} .
$$

Suppose $R=\left\{r_{1}, r_{2}, \cdots\right\}$ and $S=\left\{s_{1}, s_{2}, \cdots\right\}$ are given as in Theorem 2. It is sufficient to construct a set of numbers $\xi$ such that $\xi$ is normal to each of the bases $r_{1}, r_{2}, \ldots$ but not normal to the bases $s_{1}, s_{2}, \cdots$.

3. Preliminaries, Let

$$
\beta_{i j}=\alpha\left(r_{i}, s_{j}\right) \quad(i, j=1,2, \cdots)
$$

where $\alpha(r, s)$ is the constant in Lemma 1 .

Put

$$
\beta_{k}=\min _{1 \leqq i, j \leqq k} \beta_{i, j}
$$

and

$$
\gamma_{k}=\max \left(r_{1}, \cdots, r_{k}, s_{1}, \cdots s_{k}\right) .
$$

We may assume $\beta_{k}<1 / 2$. Put $\phi(1)=1$ and let $\phi(k)$ be the largest natural number $\phi$ which satisfies

$$
\phi \leqq \phi(k-1)+1, \quad \beta_{\phi} \geqq \beta_{1} k^{-1 / 4}, \quad \gamma_{\phi} \leqq \gamma_{1} k .
$$

Then $\phi(1), \phi(2), \cdots$ is a nondecreasing sequence of natural numbers; in iwhich every natural number occurs. We let $r_{i}^{\prime}=r_{\phi(i)}, s_{i}^{\prime}=s_{\phi(i)}$, then $\left\{r_{i}^{\prime}\right\}$ and $\left\{s_{i}^{\prime}\right\}$ have the same properties as $\left\{r_{i}\right\}$ and $\left\{s_{i}\right\}$ but further

$$
\beta_{k}^{\prime} \geqq \beta_{1}^{\prime} k^{-1 / 4} \text { and } \gamma_{k}^{\prime} \leqq \gamma_{1}^{\prime} k \text {. }
$$

Therefore we may assume that the original sequence satisfies

$$
\beta_{k} \geqq \beta_{1} k^{-1 / 4}, \quad \gamma_{k} \leqq \gamma_{1} k .
$$

We write $h(m)$ for the least number $h$, such that

$$
m \neq \equiv 0\left(\bmod 2^{h}\right) \text {. }
$$

Put $s(m)=s_{h(m)}$. Then every term $s_{i}$ occurs infinitely many times in the sequence $s(m)$.

Let $\delta_{1}, \delta_{2}, \cdots$ denote absolute constants.

4. Construction of a set of nonnormal numbers. We construct sets

$$
J_{0}=[0,1] \supset J_{1} \supset J_{2} \supset \cdots
$$


(each the union of closed intervals) as follows:

Let

$$
f(m)=e^{\sqrt{m}}+2 s_{1} m^{3} .
$$

Put

$$
\langle m\rangle=\lceil f(m)\rceil, \quad\langle m ; x\rangle=\lceil\langle m\rangle / \log x\rceil,
$$

where $\lceil x\rceil$ denotes the least integer greater than or equal to $x$,

$$
\begin{gathered}
b_{m}=\langle m+1 ; s(m)\rangle \\
a_{m+1}=\left[\frac{b_{m} \log s(m)}{\log s(m+1)}\right]+2 .
\end{gathered}
$$

Then

$$
\frac{\langle m+1\rangle}{\log s(m+1)}+2 \leqq a_{m+1} \leqq \frac{\langle m+1\rangle}{\log s(m+1)}+\log \log m+3
$$

and

$$
e^{\langle m\rangle} s(m)^{2} \leqq s(m)^{a_{m}} \leqq e^{\langle m\rangle} s(m)^{\log \log m+3} .
$$

The numbers $a_{m}$ and $b_{m}$, defined in (4) and (5), are chosen so that

$$
s(1)^{b_{1}}<s(2)^{a_{2}}<s(2)^{b_{2}}<s(3)^{a_{3}}<s(3)^{b_{3}}<\cdots .
$$

Let $J_{1}$ be the union of the intervals $I$, each of length $s(1)^{-b_{1}}$, whose left end points are of the form

$$
\xi_{1}=\frac{\varepsilon_{1}}{s(1)}+\frac{\varepsilon_{2}}{s(1)^{2}}+\cdots+\frac{\varepsilon_{b_{1}}}{s(1)^{b_{1}}}
$$

where $\varepsilon_{i}$ range over $0,1, \cdots, s(1)-2$ if $s(1)$ is odd, and over $0,1, \cdots, s(1)-3$ if $s(1)$ is even.

Put

$$
\begin{array}{rlll}
\delta(i)=2 & \text { if } & s(i) & \text { is odd } \\
=3 & \text { if } & s(i) & \text { is even } .
\end{array}
$$

There are $(s(1)-\delta(1))^{b_{1}}$ such intervals $I$ of $J_{1}$.

Suppose that $J_{k}$ has been constructed and that $I_{k}$ is an interval of $J_{k}$ of length $s(k)^{-b_{k}}$.

By (5)

$$
s(k+1)^{-a_{k+1}+2} \leqq s(k)^{-b_{k}} .
$$

Thus in each interval $I_{k}$ there are at least 


$$
\left[\frac{s(k+1)^{a_{k+1}}}{s(k)^{b_{k}}}\right]-2 \text { intervals } I_{k}^{\prime} \text { of length }
$$

$s(k+1)^{-a_{k+1}}$ whose left end points are finite "decimals" of length $a_{k+1}$ in base $s(k+1)$.

To construct $J_{k+1}$ we proceed as follows:

Let $\rho_{k}$ be the left end point of an interval $I_{k}^{\prime}$. We construct subintervals of $I_{k}^{\prime}$ of length $s(k+1)^{-b_{k+1}}$ whose left end points are of the form

$$
\xi_{k+1}=\rho_{k}+\frac{\varepsilon_{1}}{s(k+1)^{a_{k+1}+1}}+\cdots+\frac{\varepsilon_{t_{k+1}}}{s(k+1)^{b_{k+1}}}
$$

where $t_{k}=b_{k}-a_{k}$ and $\varepsilon_{1}, \cdots, \varepsilon_{t_{k+1}}$ can range over $0,1, \cdots, s(k+1)-$ $\delta(k+1)$.

In each interval $I_{k}^{\prime}$ there are $(s(k+1)-\delta(k+1)+1)^{t_{k+1}}$ such intervals. Let $J_{k+1}$ be the union of all such intervals taken over all $I_{k}^{\prime}$. Then $J_{k+1}$ is the union of at least

$$
\left(\left[\frac{s(k+1)^{a_{k+1}}}{s(k)^{b_{k}}}\right]-2\right)(s(k+1)-\delta(k+1)+1)^{t_{k+1}}
$$

intervals of length $s(k+1)^{-b_{k+1}}$. This completes the construction of the sequence of sets $J_{0} \supset J_{1} \supset \cdots$.

Lemma 2. If $\xi \in \bigcap_{i=1}^{\infty} J_{i}$ then $\xi$ is nonnormal to each base $s_{1}, s_{2}, \cdots$.

Proof. Fix $h$ and let $s=s_{h}$. Let $q$ be so large that

$$
\left(\frac{s-1}{s}\right)^{q}<2^{-h}
$$

For a number $M$ with $h(M)=h$ there are at least

$$
\sum_{\substack{m \leq M \\ h(m)=h}}\left(t_{m}-1-q\right)
$$

$q$-blocks $\varepsilon_{i+1}, \cdots, \varepsilon_{i+q}$, consisting of the digits $0,1, \cdots, s-2$ in the expansion of $\xi$, such that $i+q \leqq b_{M}$. Now $h(m)=h$ precisely if $m \equiv 2^{h-1}\left(\bmod 2^{h}\right)$. If $h(m)=h$ and $m>2^{h-1}$, then, by (6),

$$
t_{m}-1-q \geqq 2^{-h} \sum_{j=m-2^{h}+1}^{m}[(\langle j+1 ; s\rangle-\langle j ; s\rangle)-\log \log m-5-q]
$$

since $t_{m}=b_{m}-a_{m}$ and $\langle m+1 ; s\rangle-\langle m ; s\rangle$ is a nondecreasing function of $m$.

Thus (11) is at least 


$$
\begin{aligned}
\sum_{\substack{m \leq M \\
h(m)=h}} 2^{-h} & \sum_{j=m-2^{h}+1}^{m}((\langle j+1 ; s\rangle)-(\langle j ; s\rangle)-\log \log m-5-q) \\
& \geqq 2^{-h}(\langle M+1 ; s\rangle-\langle 1 ; s\rangle)-M(\log \log M+5+q) \\
& =2^{-h} b_{M}(1+0(1)) .
\end{aligned}
$$

If $\xi$ were normal to the base $s=s_{h}$, the number of $q$-blocks with digits $0,1, \cdots, s-2$ and indices smaller than $b_{M}$ would be asymptotic to $((s-1) / s)^{q} b_{M}$. By $(10)$ this is clearly not the case and Lemma 2 is proved.

5. Construction of a set of normal numbers. We also have to ensure that the numbers we have constructed are also all normal to every base from $R$. To do this we will modify our construction by discarding certain of intervals of $J_{i}$ at each stage, to obtain a new sequence, $K_{1} \supset K_{2} \supset \cdots$, with $K_{i} \subset J_{i}$.

Consider the intervals $I_{m-1}^{\prime}$. In each such interval there are $(s(m)-\delta(m)+1)^{t_{m}}$ intervals of $J_{m}$ whose left end points we denote by $\xi_{m}$.

Let

$$
A_{m}(x)=\sum_{\substack{t=-m \\ t \neq 0}}^{m} \sum_{i=1}^{m}\left|\sum_{j=\left\langle m ; r_{i}\right\rangle+1}^{\left\langle m+1 ; r_{i}\right\rangle} e\left(r_{i}^{j} t x\right)\right|^{2},
$$

where $e(x)$ denotes $e^{2 \pi i x}$.

LEMMA 3. If $m \geqq \delta_{1}$ there are at least $(s(m)-3)^{t_{m}}$ numbers $\xi_{m} \in I_{m-1}^{\prime}$ for which

$$
A_{m}\left(\xi_{m}\right) \leqq \delta_{2} m^{2}(\langle m+1\rangle-\langle m\rangle)^{2-\beta_{m} / 2} .
$$

Here $\delta_{1}$ and $\delta_{2}$ are absolute constants.

Proof. Now

$$
\sum_{\xi_{m} \in I_{m-1}^{\prime}} A_{m}\left(\xi_{m}\right)=\sum_{\substack{t=-m \\ t \neq 0}}^{m} \sum_{i=1}^{m} \sum_{\xi_{m} \in I_{m-1}^{\prime}}\left|\sum_{j=\left\langle m, r_{i}\right\rangle+1}^{\left\langle m+1 ; r_{i}\right\rangle} e\left(r_{i}^{j} t \xi_{m}\right)\right|^{2}
$$

and the inner sum,

$$
\begin{aligned}
\sum_{\xi_{m} \in I_{m-1}^{\prime}} & =\sum_{\xi_{m}} \sum_{j=\left\langle m ; r_{i}\right\rangle+1}^{\left\langle m+1 ; r_{i}\right\rangle} \sum_{g=\left\langle m ; r_{i}\right\rangle+1}^{\left\langle m+1 ; r_{i}\right\rangle} e\left(\left(r_{i}^{j}-r_{i}^{g}\right) t \xi_{m}\right) \\
& =\sum_{j} \sum_{g} \sum_{\xi_{m}} e\left(\left(r_{i}^{j}-r_{i}^{g}\right) t \xi_{m}\right) .
\end{aligned}
$$

Thus 


$$
\begin{aligned}
& \left|\sum_{\xi_{m} \in I_{m-1}^{\prime}}\right| \\
& \quad \leqq \sum_{j} \sum_{g} \prod_{k=a_{m}+1}^{b_{m}}\left|1+e\left(\frac{t\left(r_{i}^{j}-r_{i}^{g}\right)}{s(m)^{k}}\right)+\cdots+e\left(\frac{t\left(r_{i}^{j}-r_{i}^{g}\right)(s(m)-\delta(m))}{s(m)^{k}}\right)\right| .
\end{aligned}
$$

Thus

$$
\left|\sum_{\xi_{m} \in I_{m-1}^{\prime}} A_{m}\left(\xi_{m}\right)\right| \leqq \sum_{t} \sum_{i} \sum_{j} \sum_{g} \prod_{k=a_{m}+1}^{b_{m}}|1+\cdots| .
$$

We write $B_{m}(x)$ for that part of $A_{m}(x)$ for which either $|j-g|<m$ or $g$ is at least $\left\langle m+1 ; r_{i}\right\rangle-m$ and we write $C_{m}(x)$ for the remaining part.

Then

$$
A_{m}(x)=B_{m}(\dot{x})+C_{m}(x)
$$

We have the following trivial estimate.

$$
\begin{aligned}
B_{m}(x) & \leqq 10 m^{2} \sum_{i=1}^{m}\left(\left\langle m+1 ; r_{i}\right\rangle-\left\langle m ; r_{i}\right\rangle\right) \\
& \leqq \delta_{3} m^{3}(\langle m+1\rangle-\langle m\rangle) \\
& \leqq \delta_{4} m^{2}(\langle m+1\rangle-\langle m\rangle)^{2-\beta_{m}}
\end{aligned}
$$

Thus

$$
\sum_{\xi_{m}} B_{m}\left(\xi_{m}\right) \leqq \delta_{4} m^{2}(\langle m+1\rangle-\langle m\rangle)^{2-\beta_{m}}(s(m)-\delta(m)+1)^{t_{m}}
$$

Here the $\delta_{i}$ are absolute constants.

We now estimate $\sum_{\xi_{m}} C_{m}\left(\xi_{m}\right)$.

That part of the sum (12) corresponding to $C_{m}\left(\xi_{m}\right)$ is at most

$$
2 \sum_{t} \sum_{i} \sum_{g=\left\langle m ; r_{i}\right\rangle+1}^{\left\langle m+1 ; r_{i}\right\rangle} \sum_{j=g+m}^{\left\langle m+1 ; r_{i}\right\rangle-m} \prod_{k}\left|\sum_{l=0}^{s(m)-\delta(m)}\left(e\left(l t r_{i}^{g}\left(r_{i}^{j-g}-1\right) s(m)^{-k}\right)\right)\right|,
$$

since $\left|\Sigma_{x} e(x)\right|=\left|\Sigma_{x} e(-x)\right|$. By making a change of variable we obtain

$$
\left|\sum_{\xi_{m}} C_{m}\left(\xi_{m}\right)\right| \leqq 2 \sum_{t=-m}^{m} \sum_{i=1}^{m} \sum_{g=m}^{\alpha_{m}} \sum_{j=1}^{\alpha_{m}-g} \prod_{\substack{b_{m} \\ b_{m}+1}}^{b_{m}}|D(m, t, i, g, j, k)|,
$$

where

$$
\alpha_{m}=\left\langle m+1 ; r_{i}\right\rangle-\left\langle m ; r_{i}\right\rangle-m
$$

and

$$
|D|=\left|\sum_{l=0}^{s(m)-\delta(m)} e\left(t\left(r_{i}^{g}-1\right) r_{i}^{\left\langle m, r_{i}\right\rangle} \boldsymbol{r}_{i}^{j} l s(m)^{-k}\right)\right|
$$




$$
\begin{aligned}
& \leqq \frac{1}{2}(s(m)-\delta(m)+1)\left|1+e\left(t\left(r_{i}^{g}-1\right) r_{i}^{\left\langle m, r_{i}\right\rangle} r^{j} s(m)^{-k}\right)\right| \\
& =(s(m)-\delta(m)+1)\left|\cos \left(\pi L_{i} r_{i}^{j} s(m)\right)^{-k}\right|
\end{aligned}
$$

where $L_{i}=\left(r_{i}^{g}-1\right) r_{i}^{\left\langle m ; r_{i}\right\rangle} t$.

Fix $L=L_{i}, t, r=r_{i}, s=s(m), \delta=\delta(s)$ and $g$. Then the inner sum in (14) is

$$
\leqq \sum_{j=1}^{\langle m+1, r\rangle-\langle m, r\rangle-m-g} \prod_{k=a_{m}+1}^{b_{m}}\left|\cos \left(\pi L r^{j} s^{-k}\right)\right| .
$$

Now

$$
\begin{aligned}
L r^{j} s^{-b} m & \leqq r^{\langle m+1 ; r\rangle-\langle m ; r\rangle-m-g} m r^{\langle m, r\rangle} r^{g} s^{-b_{m}} \\
& =r^{\langle m+1 ; r\rangle} r^{-m} m s^{-\langle m+1 ; s\rangle} \\
& \leqq r^{\langle m+1\rangle / \log r} r^{1-m} m s^{-\langle m+1\rangle / \log s} \\
& \left.=m r^{1-m} \leqq 1 / 2 \quad \text { (provided } m>1, r^{\prime} \geqq 4\right) .
\end{aligned}
$$

Thus

$$
\prod_{k=b_{k+1}}^{\infty}\left|\cos \left(\pi L r^{j} s^{-k}\right)\right| \geqq \prod_{k=1}^{\infty}\left|\cos \left(\pi / 2^{k+1}\right)\right|=\delta_{5}>0 .
$$

The sum (15) is at most equal to

$$
\delta_{6} \sum_{j=1}^{\langle m+1 ; r\rangle-\langle m ; r\rangle-m-g} \prod_{k=a_{m}+1}^{\infty}\left|\cos \left(\pi L r^{j} / s^{k}\right)\right| \text {. }
$$

Now

$$
\begin{aligned}
|L| & \geqq\left(r^{m}-1\right) r^{\langle m ; r\rangle} \geqq\left(r^{m}-1\right) e^{\langle m\rangle} \\
& \geqq\left(r^{m}-1\right) s(m)^{a_{m}} s(m)^{-\log \log m-3} \quad \text { by }(6) \\
& \geqq s(m)^{a_{m}+1}
\end{aligned}
$$

provided

$$
r^{m} \geqq s(m)^{\log \log m+4}+1,
$$

which holds for $m$ sufficiently large, by (2). Hence from $m \geqq \delta_{4}$ we may apply Lemma 1 and see that (15) is at most

$$
2 \delta_{6}(\langle m+1 ; r\rangle-\langle m ; r\rangle)^{1-\alpha(r, s)} \text {. }
$$

Thus we have

$$
\left|\sum_{\xi_{m} \in I_{m-1}^{\prime}} C_{m}\left(\xi_{m}\right)\right| \leqq \delta_{7} m^{2}(\langle m+1\rangle-\langle m\rangle)^{2-\beta m}(s-\delta+1)^{t_{m}}
$$

Combining this with the estimate for $\left|\sum B_{m}\left(\xi_{m}\right)\right|$ we have

$$
\left|\sum_{\xi_{m} \in I_{m-1}^{\prime}} A_{m}\left(\xi_{m}\right)\right| \leqq \delta_{2} m^{2}(\langle m+1\rangle-\langle m\rangle)^{2-\beta_{m}}(s-\delta+1) \text {. }
$$


Hence the number of $\xi_{m} \in I_{m-1}^{\prime}$ for which

$$
A_{m}\left(\xi_{m}\right)>\delta_{2} m^{2}(\langle m+1\rangle-\langle m\rangle)^{2-\beta_{m} / 2}
$$

is at most

$$
(\langle m+1\rangle-\langle m\rangle)^{-\beta_{m} / 2}(s-\delta+1)^{t_{m}}
$$

But

$$
\beta_{m} \geqq \beta_{1} m^{-1 / 4} \quad \text { and } \quad(\langle m+1\rangle-\langle m\rangle) \geqq \frac{e^{\sqrt{m}}}{2 \sqrt{m+1}}
$$

and so

$$
\begin{aligned}
(\langle m+1\rangle-\langle m\rangle)^{-\beta_{m} / 2} & \leqq\left(\frac{2 \sqrt{m+1}}{e^{\sqrt{m}}}\right)^{\beta_{1} m^{-1 / 4} / 2} \\
& =\left[(2 \sqrt{m+1})^{m^{-1 / 4}} e^{m^{1 / 4}}\right]^{\beta_{1} / 2} \\
& <1 / 2 \text { for } m>\delta_{4} .
\end{aligned}
$$
which

Hence there are at least $\frac{1}{2}(s-\delta+1)^{t_{m}}$ numbers $\xi_{m} \in I_{m-1}^{\prime}$ for

$$
A_{m}\left(\xi_{m}\right) \leqq \delta_{2} m^{2}(\langle m+1\rangle-\langle m\rangle)^{2-\beta_{m} / 2} .
$$

For $m \geqq \delta_{1}(s-3)^{t_{m}}<\frac{1}{2}(s-\delta+1)^{t_{m}}$ and the proof of Lemma 3 is complete.

We construct a sequence of sets $K_{1} \supset K_{2} \supset \cdots$ in the same way as $J_{1} \supset J_{2} \supset \cdots$ was constructed. But at each stage in our construction of $\left\{K_{m}\right\}$ we use only the $(s(m)-3)^{t_{m}}$ points $\xi_{m}$ satisfying Lemma 3.

Lemma 4. If $\xi \in \bigcap_{m=1}^{\infty} K_{m}$ then

$$
A_{m}(\xi) \leqq \delta_{8} m^{2}(\langle m+1\rangle-\langle m\rangle)^{2-\beta_{m} / 2} .
$$

Proof. Clearly

$$
\begin{aligned}
& A_{m}(\xi)=A_{m}(\xi)-A_{m}\left(\xi_{m}\right)+A_{m}\left(\xi_{m}\right) \text { and } \\
& A_{m}(\xi)-A_{m}\left(\xi_{m}\right)=C_{m}(\xi)-C_{m}\left(\xi_{m}\right)+B_{m}(\xi)-B_{m}\left(\xi_{m}\right) .
\end{aligned}
$$

We estimate $B_{m}(\xi)-B_{m}\left(\xi_{m}\right)$ as we did for $B_{m}(x)$ above.

Put $L_{g}=\left(r^{g}-1\right) r^{\langle m+1 ; r\rangle-m-g} t\left(\xi-\xi_{m}\right)$. Then $\left|L_{g}\right| \leqq 1 / 2$ for $m \geqq \delta_{1}$. The part of the expression for $\left|C_{m}(\xi)-C_{m}\left(\xi_{m}\right)\right|$ for which $t$ and $r=r_{i}$ remain fixed is at most equal to

$$
2 \sum_{g=1}^{\alpha_{m}} \sum_{j=1}^{\alpha_{m}-g}\left|e\left(L_{g} r^{-j}\right)-1\right|
$$




$$
\begin{aligned}
& \leqq 2 \sum_{g=1}^{\langle m+1 ; r\rangle-\langle m ; r\rangle-m} \sum_{j=1}^{\infty} r^{-j} \\
& \langle 2(\langle m+1 ; r\rangle-\langle m ; r\rangle) .
\end{aligned}
$$

Thus

$$
\begin{aligned}
\left|C_{m}(\xi)-C_{m}\left(\xi_{m}\right)\right| & \leqq \delta_{9} m^{3}(\langle m+1\rangle-\langle m\rangle) \\
& \leqq \delta_{10} m^{2}(\langle m+1\rangle-\langle m\rangle)^{2-\beta_{m} / 2}
\end{aligned}
$$

Thus

$$
\left|A_{m}(\xi)-A_{m}\left(\xi_{m}\right)\right| \leqq \delta_{11} m^{2}(\langle m+1\rangle-\langle m\rangle)^{2-\beta_{m} / 2}
$$

and so, combining this with Lemma 3,

$$
A_{m}(\xi) \leqq \delta_{8} m^{2}(\langle m+1\rangle-\langle m\rangle)^{2-\beta_{m} / 2} .
$$

We now apply the following lemma, Hilfsatz 8, of Schmidt [3] to show that $\xi$ is normal to every base from $R$.

Lemma 5. If $A_{m}(\xi) \leqq \delta_{8} m^{2}(\langle m+1\rangle-\langle m\rangle)^{2-\beta_{m} / 2}$ for $m \geqq \delta_{4}$ then $\xi$ is normal to each base $r_{1}, r_{2}, \cdots$.

Thus if $K=\bigcap_{m=1}^{\infty} K_{m}$, then $K$ is a set of numbers inormal to every base from $R$ and to no base from $S$. It remains to estimate the Hausdorff dimension of $K$.

6. Estimation of the Hausdorff dimension of $K, K_{m}$ is a linear set consisting of

$$
N_{m}=\prod_{k=1}^{m}(s(k)-3)^{b_{k}-a_{k}}\left(\left[\frac{s(k)^{a_{k}}}{s(k-1)^{b_{k-1}}}\right]-2\right)
$$

intervals of length $s(m)^{-b_{m}}=\delta_{m}$.

Hence

$$
N_{m}>\prod_{k=1}^{m}(s(k)-3)^{b_{k}-a_{k}}
$$

Now

$$
\begin{aligned}
(s-3)^{n} & =s^{(\log (s-3) / \log s) \cdot n} \geqq s^{(\log (A-3) / \log A) \cdot n}, \quad(\text { if } s \geqq A), \\
& =e^{n(\log (A-3) / \log A) \cdot \log s} .
\end{aligned}
$$

Thus

$$
N_{m}>\exp \left[\frac{\log (A-3)}{\log A} \sum_{k=1}^{m}\left(b_{k}-a_{k}\right) \log s(k)\right]
$$




$$
\begin{aligned}
& \geqq \exp \left[\frac{\log (A-3)}{\log A} \sum_{k=1}^{m}\langle k+1\rangle-\langle k\rangle-(\log s(k))(\log \log k+3)\right] \\
& \geqq \exp \left[\frac{\log (A-3)}{\log A}\langle m+1\rangle(1+O(1))\right] .
\end{aligned}
$$

We also have

$$
\frac{\delta_{m-1}}{\delta_{m}}=\frac{s(m)^{b_{m}}}{s(m-1)^{b_{m-1}}} \leqq s(m) e^{\langle m+1\rangle-\langle m\rangle} \leqq \exp \left(\frac{\langle m+1\rangle}{\sqrt{m}}+\log s(m)\right)
$$

and

$$
\delta_{m}^{t}=s(m)^{-b_{m} t} \geqq \exp (-t\langle m+1\rangle)
$$

Thus

$$
\begin{aligned}
\sum_{m} & \frac{\delta_{m-1}}{\delta_{m}}\left(N_{m} \delta_{m}^{t}\right)^{-1} \\
& \leqq \sum_{m} \exp \left[\frac{\langle m+1\rangle}{\sqrt{m}}+\log s(m)-\frac{\log (A-3)}{\log A}\langle m+1\rangle(1+O(1))+t\langle m+1\rangle\right] \\
\quad & \sum_{m} \exp \left[\langle m+1\rangle\left(t-\frac{\log (A-3)}{\log A}\right)(1+O(1))\right] .
\end{aligned}
$$

This sum will certainly converge for all $t<\log (A-3) / \log A$.

We apply the following theorem of Eggleston, [1], to estimate the Hausdorff dimension of $K$.

Theorem. Suppose $K_{k}(k=1,2, \cdots)$ is a linear set consisting of $N_{k}$ closed intervals each of length $\delta_{k}$. Let each interval of $K_{k}$ contain $m_{k+1}>0$ disjoint intervals of $K_{k+1}$.

Suppose that $0<s_{0} \leqq 1$ and that for all $s<s_{0}$ the sum

$$
\sum_{k} \frac{\delta_{k-1}}{\delta_{k}}\left(N_{k}\left(\delta_{k}\right)^{s}\right)^{-1}
$$

converges. Then $K=\bigcap_{k=1}^{\infty} K_{k}$ has dimension greater than or equal to $s_{0}$.

Clearly all the conditions necessary to apply Eggleston's theorem are satisfied where we may take $s_{0}=\log (A-3) / \log A$. This proves Theorem 2.

The author would like to thank the referee for his careful reading of the original manuscript and for his many helpful suggestions for improving the presentation of this paper. 


\section{REFERENCES}

1. H. G. Eggleston, Sets of fractional dimension which occur in some problems of number theory, Proc. London Math. Soc., 54 (1951-52), 42-93.

2. W. M. Schmidt, On normal numbers, Pacific J. Math., 10 (1960), 661-672.

3. - Über die Normalität von Zahlen zu verschiedenen Basen, Acta Arith., 7 (1961-62), 299-309.

4. On badly approximable numbers and certain games, Trans. Amer. Math. Soc., 123 (1966), 178-199.

Received November 30, 1979 and in revised form May 14, 1980.

Illinots State University

Normal, IL 61761 


\section{PACIFIC JOURNAL OF MATHEMATICS}

\section{EDITORS}

DONALD BABBITT (Managing Editor)

University of Galifornia

Los Angeles, California 90024

Hugo RossI

University of Utah

Salt Lake City, UT 84112

C. C. MOORE AND ANDREW OGG

University of California

Berkeley, CA 94720
J. DugundJI

Department of Mathematics University of Southern California Los Angeles, California 90007

R. FinN and J. Milgram Stanford University Stanford, California 94305

\section{ASSOCIATE EDITORS}

R. ARENS

E. F. BeCKenbaCh

B. H. NEUManN

F. WOLF

K. YosHIDA

\section{SUPPORTING INSTITUTIONS}

UNIVERSITY OF ARIZONA

UNIVERSITY OF BRITISH COLUMBIA

CALIFORNIA INSTITUTE OF TECHNOLOGY

UNIVERSITY OF CALIFORNIA

MONTANA STATE UNIVERSITY

UNIVERSITY OF NEVADA, RENO

NEW MEXICO STATE UNIVERSITY

OREGON STATE UNIVERSITY
UNIVERSITY OF OREGON

UNIVERSITY OF SOUTHERN CALIFONIA

STANFORD UNIVERSITY

UNIVERSITY OF HAWAII

UNIVERSITY OF TOKYO

UNIVERSITY OF UTAH

WASHINGTON STATE UNIVERSITY

UNIVERSITY OF WASHINGTON 


\section{Pacific Journal of Mathematics}

\section{Vol. 95, No. $1 \quad$ September, 1981}

John Allen Beachy and William David Blair, On rings with bounded

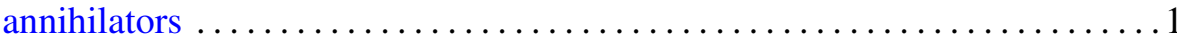

Douglas S. Bridges, A constructive look at positive linear functionals on

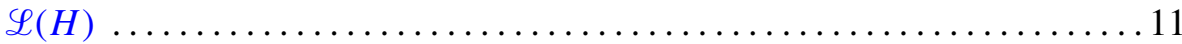

Muneo Chō and Makoto Takaguchi, Boundary points of joint numerical ranges

W. J. Cramer and William O. Ray, Solvability of nonlinear operator equations

Lester Eli Dubins and Gideon Schwarz, Equidiscontinuity of

Borsuk-Ulam functions

Maria Fragoulopoulou, Spaces of representations and enveloping 1.m.c.

*-algebras

Robert F. Geitz and J. Jerry Uhl, Jr., Vector-valued functions as families of scalar-valued functions

Ross Geoghegan, The homomorphism on fundamental group induced by a homotopy idempotent having essential fixed points

Ross Geoghegan, Splitting homotopy idempotents which have essential fixed points

Paul Jacob Koosis, Entire functions of exponential type as multipliers for

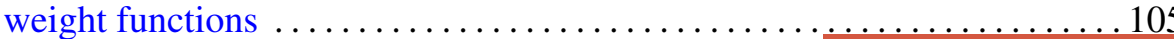

David London, Monotonicity of permanents of certain doubly stochastic matrices

Howard J. Marcum, Two results on cofibers

Giancarlo Mauceri, Zonal multipliers on the Heisenberg group

Edward Wilfred Odell, Jr. and Y. Sternfeld, A fixed point theorem in $c_{0} \quad \ldots 161$

Bernt Karsten Oksendal, Brownian motion and sets of harmonic measure zero

Andrew Douglas Pollington, The Hausdorff dimension of a set of normal numbers

Joe Repka, Base change lifting and Galois invariance ...

Gerald Suchan, Concerning the minimum of permanents on doubly stochastic circulants

Jun-ichi Tanaka, On isometries of Hardy spaces on compact abelian groups

Aaron R. Todd, Quasiregular, pseudocomplete, and Baire spaces 